\title{
A MOBILE GAIT ANALYSIS SYSTEM FOR OPTIMIZATION OF PROSTHETIC ALIGNMENTS
}

\author{
Westebbe $\mathrm{B}^{1}$, Thiele $\mathrm{J}^{1}$, Kraft $\mathrm{M}^{1}$ \\ ${ }^{1}$ Technical University Berlin, Germany \\ b.westebbe@tu-berlin.de
}

\begin{abstract}
The prosthesis alignment is of central importance for a harmonic gait, especially for upper limb amputees. Today alignment optimization is based on static measuring and the experience of the orthopaedic technician. To objectify the alignment process a mobile gait analysis system based on 10 inertial sensors and a 6 DOF force and moment sensor was developed at the TU Berlin. The dedicated software adds dynamic gait parameters into the optimization process and guides the necessary changes in the prosthesis alignment. Therefore 19 common alignment changes were analysed based on measurements with 2 subjects fitted with the C-Leg knee. The anterior and posterior displacement of the knee will be the focus of an additional study with 6 subjects.
\end{abstract}

\section{Keywords: amputee, upper limb, prostheses alignment,} optimization, mobile gait analysis

\section{Introduction}

The correct alignment of prostheses is of central importance for amputees to avoid asymmetrical stress of the musculoskeletal system [1]. Increased biomechanical stress of the residual leg can force degenerative processes in its joints [2]. Today prosthetic alignment is supported by means of static measuring systems (e.g. LASAR Posture, LASAR Assembly) which cannot enable the evaluation of all aspects of the gait. Thus a system for optimization of prosthetic alignments by dynamic parameters was developed at the TU Berlin.

\section{Methods}

Measuring system: A mobile gait analysis system for amputees has been designed by combining inertial sensor based motion tracking with the Oktapod system [3] for measuring forces and moments in 6 degrees of freedom in a lower limb prosthesis. The 10 motion trackers (MTw Wireless by Xsens) combine accelerometers, gyroscopes and magnetoscopes. Joint positions and angles are calculated based on the exploitation of kinematic constraints. The advantage of these newly developed algorithms is that they are neither depended on an exact mounting orientation of the sensors in relation to the body segments nor on exact calibration movements [4].

Data collection: With the mobile gait analysis system measurements of gait on even ground with self-selected velocity and different prosthetic alignments were performed. Overall 19 alignment variations were analyzed based on data from two subjects. Subsequently the variations knee anterior and knee posterior displacement will be further examined by additional six subjects at the Hannover Medical School. All subjects used their own socket and were fitted with the C-Leg and either the 1D35 or the 1C40 (all Otto Bock HealthCare). For validation purposes all measurements were conducted in a gait lab (Vicon, System 460, M-Cam; Kistler, Typ 9287A).

Data processing: The collected data of the different sources is synchronized and physical properties like joint angles, joint moments, energy expenditure and the load line are calculated. Inertial sensors on both feet are used to detect the gait phases. Based on the forces and moments measured in the prosthesis a step detection and filter has been implemented. The filter is necessary to avoid that braking and accelerating steps are included in the further data processing.

\section{Results}

The collected gait data varies significantly between subjects. This is shown in Figure 1 where the measured sagittal moments of the bench alignment based on manufacturers' instructions, the knee anterior and posterior displacement are compared. Subject A performs stance phase flexion with the bench alignment and the alignment with knee displaced anteriorly shown by the negative sagittal moments that cause knee flexion. Subject B suppresses this shock absorbing movement actively, which can be explained by a high need for safety while walking.

Additionally in Figure 1 the expected moments for the two alignment variations are shown, which are calculated from the bench alignment data by adding the torque that results from the additional lever when the knee centre is shifted. Thereby compensation strategies of the amputee can be observed. The expected knee flexion moments while displacing the prosthetic knee anteriorly were not reached by subject $A$ in the first part of the gait cycle $(0-30 \%$ gc, ant measured). The expected moments would cause an exaggerated stance phase flexion. That would lead to a high energy expenditure why it is avoided by active compensation with the amputees stump. For subject B this compensation strategy is not necessary as the sagittal moments are positive with every alignment in the first part of the stance phase $(0-30 \% \mathrm{gc}$, ant measured). Both subjects show, that the potentially accelerated knee flexion in the pre-swing phase $(50-60 \% \mathrm{gc}$, ant expected) shown by the expected high knee flexion moment is avoided to proper initiation of the swing phase flexion.

The collected data showed not only inter-subject variability but also significant differences depending on the physical and mental state on the day of testing. Measurements of the same alignment on different days often showed stronger distinctions than those of the alignment variations. 

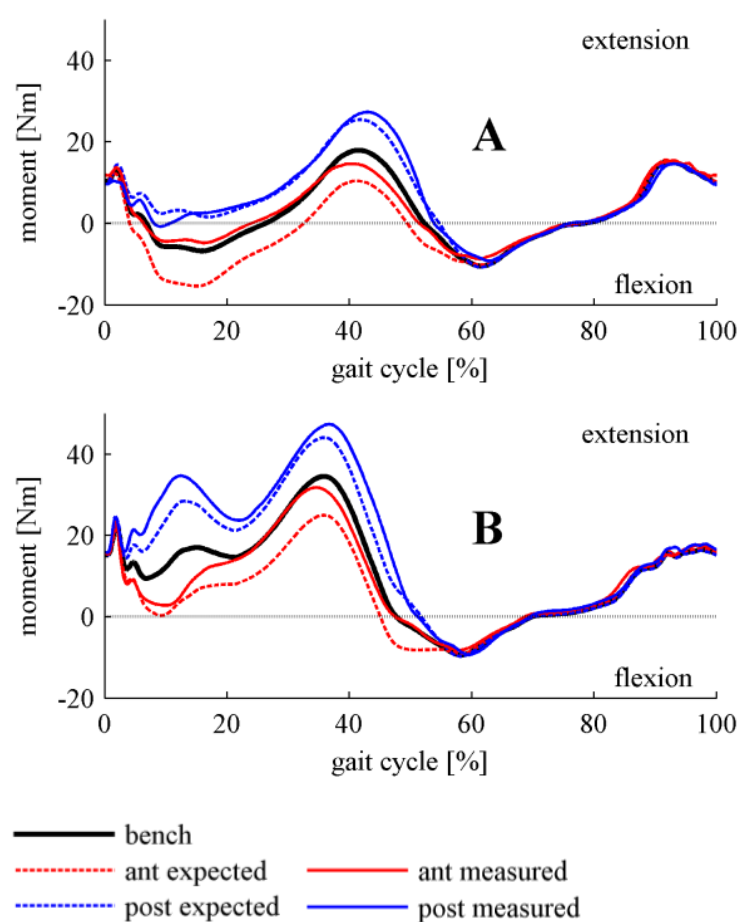

Figure 1: Comparison of the measured and the calculated sagittal moment for bench alignment, knee anterior and posterior displacement of subject $\mathrm{A}$ and $\mathrm{B}$

\section{Discussion}

When developing the expert system it is necessary to build a knowledge base that defines which change on the alignment has positive effects and which disturbs harmonic movements. The results show that it is not possible to define an absolute optimum for every parameter (Table 1), although some variables are useable for optimization without further processing. For those parameters that have no absolute optimum it is necessary to define a zero level for each patient as a starting point for the optimization process. This can either be a measurement with the bench alignment or one with the actual alignment that the patient is used to. After the baseline measurement, alignment variations are performed, following recommendations of this expert system and the experience of the orthopaedic technician.

Table 1: Examplary optimization parameters

\begin{tabular}{lll}
\hline parameter & adjustment & optimum \\
\hline Energy consumption & Minimize & Relative \\
Compensation mechanisms & Minimize & Absolute \\
Gait symmetry & Maximize & Absolute \\
Inter-step-variance & Minimize & Absolute \\
Torsion & Minimize & Relative \\
Stance phase flexion & Maximize & Absolute
\end{tabular}

In the first version of the expert system, recommendations for knee displacement in anterior and posterior direction are given. Therefore no sophisticated statistical training is needed to implement a robust decision-making ability of the system. Compensation effects that for example occur if an alignment feels insecure can disturb the optimization process and have to be detected properly. In order to be able to decide if extensive compensation effects appeared, the expert system has to know which change in the alignment has been made to calculate the difference between the expected and actual values. If the compensation is predominating, not an alignment change will be recommended but an adjustment in patient behaviour.

The final decision if the alignment respectively a parameter is fully optimized has to be made by the orthopaedic technician since the functional capability of each patient cannot be described comprehensively to the system (Figure 2).

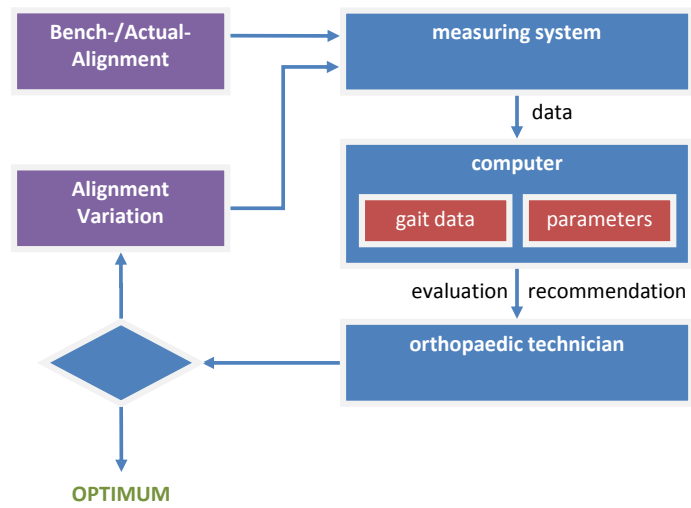

Figure 2: Flowchart of expert system

In the future it is planned to expand the expert system to give recommendations for other alignment optimization possibilities beside knee displacement in anterior and posterior direction. Also online data processing is in development to accelerate user feedback for patients and orthopaedic technicians.

\section{Acknowledgement}

This work is supported by the German Federal Ministry of Education and Research (BMBF) within the cooperative research project mebGO (grant 13EZ1112A) and the Otto Bock HealthCare GmbH.

\section{Bibliography}

[1] L. Fang, X. Jia, and R. Wang, "Modeling and simulation of muscle forces of trans-tibial amputee to study effect of prosthetic alignment," Clin Biomech (Bristol, Avon), vol. 22, no. 10, pp. 1125-1131, 2007.

[2] R. Gailey, K. Allen, J. Castles, J. Kucharik, and M. Roeder, "Review of secondary physical conditions associated with lower-limb amputation and long-term prosthesis use," Journal of rehabilitation research and development, vol. 45, no. 1, pp. 15-30, 2008.

[3] S. Oehler, M. Kraft, and M. Pusch, "Mobilitäts- und Belastungsmessungen an Oberschenkelamputierten Ergebnisse einer einjährigen Studie," Biomedizinische Technik/Biomedical Engineering, vol. 55, no. S1, pp. 63-66, 2010.

[4] T. Seel, T. Schauer, and J. Raisch, "Joint axis and position estimation from inertial measurement data by exploiting kinematic constraints," in IEEE MultiConference on Systems and Control, 2012, pp. 45-49. 